\title{
Long-term progression-free survival in a case of hepatocellular carcinoma with vertebral metastasis treated with a reduced dose of sorafenib: Case report and review of the literature
}

\author{
JUAN DU, XIAOPING QIAN and BAORUI LIU \\ The Comprehensive Cancer Center of Drum Tower Hospital, Medical School of Nanjing University \\ and Clinical Cancer Institute of Nanjing University, Nanjing 210008, P.R. China
}

Received July 15, 2012; Accepted October 9, 2012

DOI: $10.3892 / \mathrm{ol} .2012 .974$

\begin{abstract}
Hepatocellular carcinoma (HCC) is the most common primary cancer of the liver. Prognosis and treatment options are stage-dependent. Typically, prognosis of patients with unresectable HCC is poor, particularly for patients with distant metastasis. Sorafenib has demonstrated an overall survival benefit and has become the new standard of care for advanced HCC. However, in metastatic HCC, long-term progression-free survival for five years with reduced doses of sorafenib is extremely rare. In clinical practice, certain patients are discontinuing the use of this drug due to its side-effects. We highlight the importance of prolonged sorafenib administration, even at reduced doses. We describe an unusual case of a 74-year-old patient with HCC metastatic to the vertebrae that responded to a reduced dose of sorafenib and has subsequently demonstrated no signs of disease progression since starting treatment almost five years ago. This suggests that certain patients with highly progressive HCC involving bone metastasis may achieve long-term survival by reduced doses of sorafenib.
\end{abstract}

\section{Introduction}

Hepatocellular carcinoma (HCC), which accounts for $90 \%$ of all primary liver cancers, is the fifth most common malignancy worldwide and the third most common cause of cancer-related mortality globally (1). Due to its asymptomatic nature, early HCC is difficult to detect and numerous patients present with advanced or unresectable forms of the disease at diagnosis. Thus, the prognosis for such patients remains poor. Previously, the treatment of advanced HCC with conventional

Correspondence to: Professor Baorui Liu, The Comprehensive Cancer Center of Drum Tower Hospital, Zhongshan Road 321\#, Nanjing, 210008, Jiangsu, P.R. China

E-mail: baoruiliu@nju.edu.cn

Key words: hepatocellular carcinoma, sorafenib, long-term survival, molecular targeted drug antineoplastic drugs has not resulted in satisfactory outcomes, whilst the mean survival time of an untreated HCC patient is 7-8 months (2). Sorafenib (Nexavar ${ }^{\circledR}$, Bayer Healthcare Pharmaceuticals, USA) is an oral multikinase inhibitor that mainly targets Raf kinases, vascular endothelial growth factor receptors 1, 2 and 3, and platelet-derived growth factor receptor beta. It has been demonstrated to improve overall survival in patients with advanced HCC in two randomized, double-blinded, placebo-controlled trials $(3,4)$. This drug has been approved as the first-line therapy for such patients (5). Observations of the tumor response and its clinical course under treatment with sorafenib were markedly different from those of conventional cytotoxic agents. Notably, the majority of patients who responded to sorafenib exhibited stable disease (SD) in both of the aforementioned studies, and sorafenib seldom induced the dimensional tumor shrinking typically observed with conventional cytotoxic agents. Therefore, it has been suggested that sorafenib prolongs survival by delaying disease progression. However, long-term progression-free survival for almost five years with a reduced dose of sorafenib in metastatic HCC is extremely rare. We describe a case of a 74-year-old patient with hepatitis B, cirrhosis and HCC, who was treated with a reduced dose of sorafenib (200 mg twice a day) and achieved progression-free survival for almost five years. Written informed consent was obtained from the patient for publication of this case report and any accompanying images. The study was approved by the ethics committee of Drum-Tower Hospital, Nanjing, China.

\section{Case report}

A 74-year-old Chinese man who had suffered from hepatitis B virus-related cirrhosis since 1987 was referred to the Drum Tower Hospital, Nanjing, China, due to the discovery of a liver mass during an ultrasonographic examination in October 2005. Enhanced computed tomography (CT) confirmed the presence of a lesion approximately $55 \times 50 \mathrm{~mm}$ in size, located in the inferior segment of the right side of the liver. This mass was characterized by its indistinct margins and infiltration, with wash-in in arterial phase and wash-out in late phase, which are typical of HCC. The patient's alpha-fetoprotein (AFP) serum level was elevated to $120 \mathrm{ng} / \mathrm{ml}$ (normal level is 


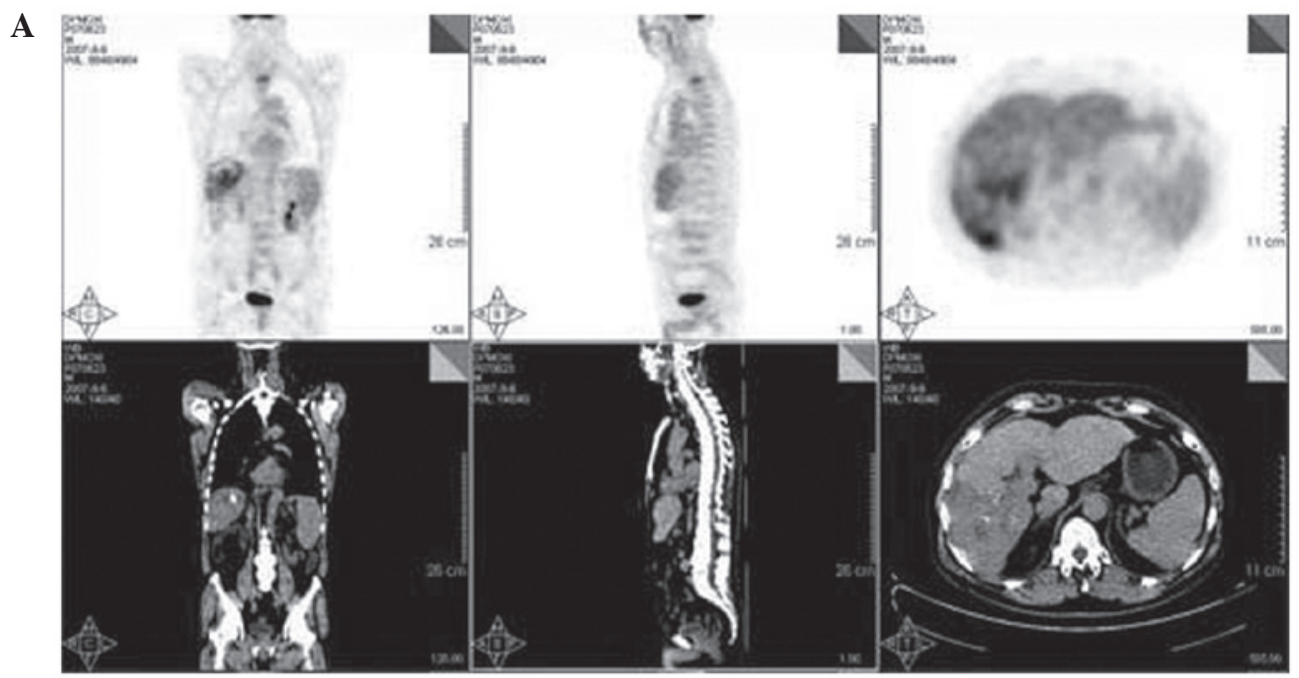

B

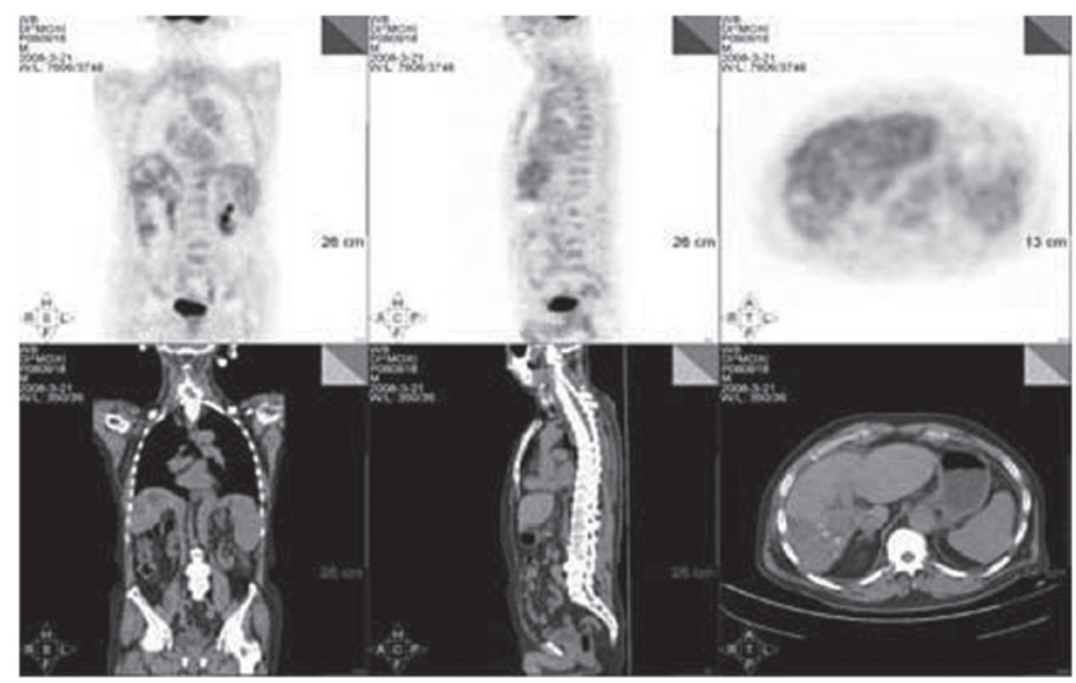

Figure 1. (A) Computed tomography with positron emission tomography (CT/PET) scan prior to commencing treatment with sorafenib. The radioactive abnormal uptake shadows are visible in the inferior segment of the right side of the liver and the second thoracic vertebra. (B) CT/PET scan six months after the start of treatment with sorafenib. The radioactive abnormal uptake shadows on the right lobe of the liver and the second thoracic vertebra have disappeared.

below $10 \mathrm{ng} / \mathrm{ml})$. The pathological examination of fine needle aspiration (FNA) confirmed the presence of a moderate differentiated hepatocellular carcinoma. The patient was otherwise healthy (ECOG PS 0) and hepatic synthesis was well-retained without clinical signs of liver impairment (Child-Pugh class A). Additionally, extrahepatic metastasis was not observed. The patient was submitted to transarterial chemoembolization in November 2005 and his AFP serum level was reduced to a normal level following treatment.

Between March 2006 and June 2007 different therapies of transarterial chemoembolization, percutaneous ethanol injection and radiofrequency ablation were conducted due to local tumor recurrence. The patient's AFP serum level returned to normal after each treatment. However, in July 2007, the patient's AFP serum level rose to $1,220 \mathrm{ng} / \mathrm{ml}$ and did not decrease following either percutaneous ethanol injection or radio frequency ablation therapy. By September 2007 , the patient's AFP serum level had increased to $22,100 \mathrm{ng} / \mathrm{ml}$. A surveillance computed tomography with positron emission tomography (CT/PET) scan revealed a radioactive abnormal uptake shadow mass of $86 \times 57 \mathrm{~mm}$ in the inferior segment of the right side of the liver that demonstrated increased uptake of FDG (average SUV, 5.5). Additionally, a radioactive abnormal uptake shadow mass was observed in the second thoracic vertebra with the same increased uptake of FDG (Fig. 1A). Magnetic resonance imaging (MRI) revealed that the second thoracic vertebra signal intensity was uneven and enhancement was visible in contrast-enhanced scans, confirming vertebral metastasis (Fig. 2).

As it was no longer possible to utilize regional therapy, treatment with sorafenib tablets at a dose of $400 \mathrm{mg}$ twice a day was initiated. The first assessment of the efficacy of the treatment regimen took place when the patient had undergone treatment for two weeks. The patient's AFP serum level had significantly decreased from 22,100 to $1,436 \mathrm{ng} / \mathrm{ml}$. However, the patient required a dose reduction of sorafenib to $200 \mathrm{mg}$ twice a day, due to a grade 3 hand-foot skin reaction, grade 2 diarrhea and significant deterioration in performance status. 


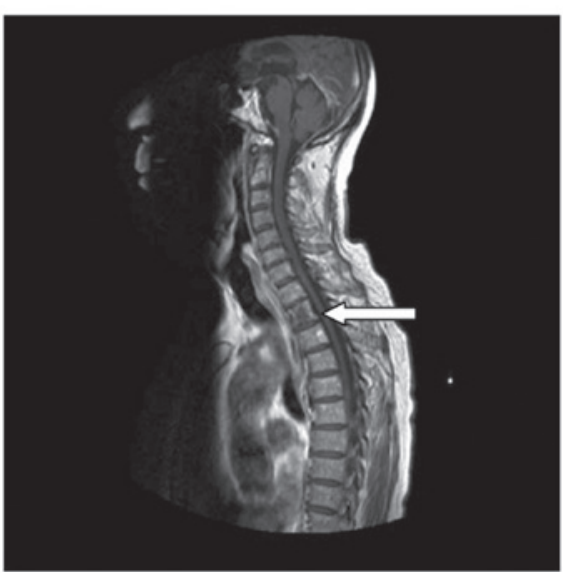

Figure 2. Contrast-enhanced magnetic resonance imaging (MRI) scans prior to commencing treatment with sorafenib. The abnormal enhancement lesion is visible in the second thoracic vertebra (white arrow).

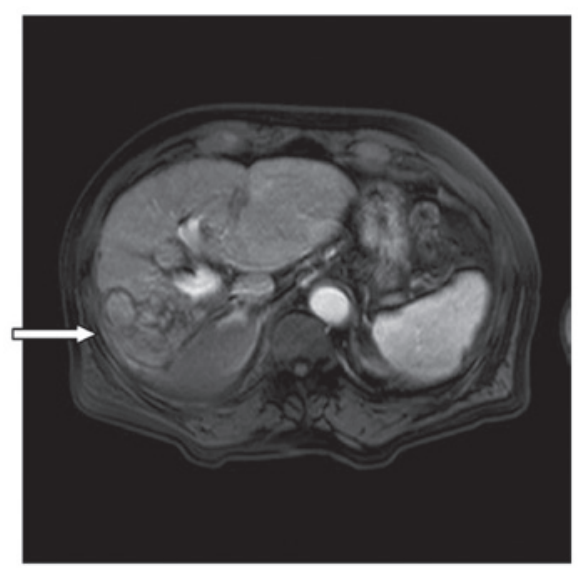

Figure 3. Contrast-enhanced magnetic resonance imaging (MRI) scans 20 months after the start of treatment with sorafenib. No hypervascular lesions are identified in the liver parenchyma tissue, not even at the level of the previous lesion on the right side of the liver (white arrow).

The second assessment took place after 2 months of treatment. The AFP serum level had decreased to within the normal range. The patient remained stable on sorafenib at a dose of $200 \mathrm{mg}$ twice a day and the main adverse side-effect was a grade 1 hand-foot skin reaction, which was simply controlled with local treatment. No other obvious adverse reactions were evident.

Following 6 months of treatment, a third assessment took place. A follow-up CT/PET scan revealed that liver radioactivity was distributed uniformly; on the right lobe, numerous round/oval radioactive distribution defects of various sizes were observed, which were considered to be tumor necrosis based on the local treatment history. The radioactive abnormal uptake shadows on the right lobe of the liver and the second thoracic vertebra had disappeared (Fig. 1B).

A fourth assessment took place after the patient had undergone treatment for 20 months. The patient's AFP serum level remained steady within the normal range. MRI revealed the presence of multiple nodules on the right lobe of the liver;

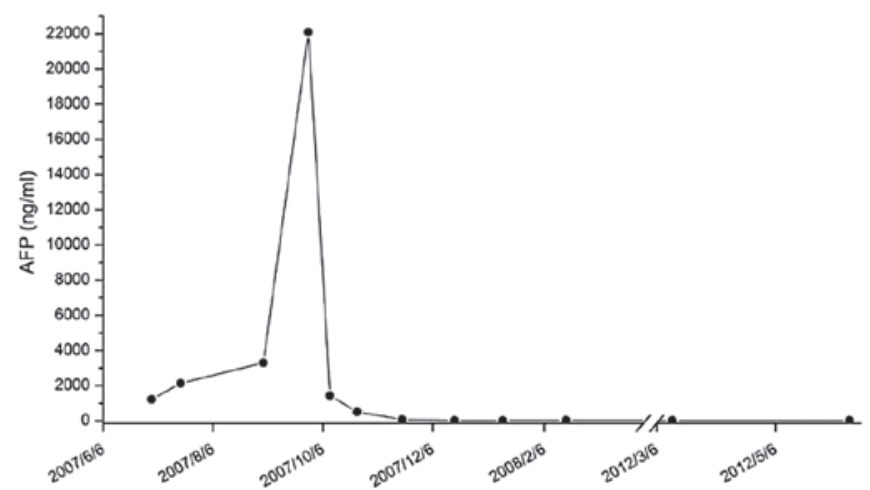

Figure 4. Analysis of alpha-fetoprotein (AFP) serum level. A clear reduction of marker serum level was observed following two weeks of treatment with sorafenib, which remained within the normal range for almost five years.

however, the contrast-enhanced scans did not identify hypervascular lesions in the liver parenchyma tissue (Fig. 3).

A fifth assessment took place after four years of treatment. The patient's AFP serum level remained within the normal range. Additionally, the contrast-enhanced MRI scans did not reveal any abnormal enhancement lesions in the whole spine.

At the last visit, in May 2012, almost five years after the diagnosis of bone metastasis, the patient's AFP serum level remained within the normal range (Fig. 4). MRI confirmed response by the liver and thoracic vertebra lesions, whilst no new lesions were found.

Until now, treatment with sorafenib has been continued and a follow-up program to evaluate the duration of the response is in progress. The patient also maintained a relatively active lifestyle and no additional adverse events have been identified.

\section{Discussion}

The present case concerns long-term progression-free survival, extensively supported by imaging and the evaluation of tumor markers, which occurred in a patient suffering from advanced HCC with bone metastasis that was treated with sorafenib.

The approval of sorafenib and the active development of numerous other molecularly targeted agents in HCC have presented challenges to understand the mechanism of action of sorafenib and to identify predictive biomarkers capable of selecting patients who are more likely to benefit from sorafenib. Raf kinase is overexpressed in a high percentage of human HCC tumors (6), and the RAF/MEK/ERK pathway can be activated by key etiologic factors including hepatitis $\mathrm{B}$ virus (HBV) and hepatitis $\mathrm{C}$ virus (HCV) infection (7). A previous study provides preliminary evidence that baseline phosphorylated extracellular signaling-regulated kinase (pERK) may be a relevant marker to reflect the level of constitutive activation of the RAF/MAPK kinase (MEK)/ ERK signaling pathway, and has the potential value to predict responses to sorafenib (8). The clinical data from the initial single-arm phase II study and the preliminary findings from 
the randomized phase III study also suggest a correlation between baseline archived tumor pERK levels and time to tumor progression in HCC patients. It is likely that baseline pERK will become a useful predictive biomarker of the response to, and the clinical benefits of, sorafenib in HCC; however, this requires validation in future large-prospective studies. The response observed in our patient was unique and the underlying mechanism is not completely understood. We hypothesize that the patient's HCC may represent a rare condition where only a single or few pathway(s) are mainly responsible for tumor formation, including the Raf or vascular endothelial growth factor (VEGF) pathway. Therefore, sorafenib, as a Raf or VEGF inhibitor, may have completely blocked such growth signals, thus generating an exceedingly optimized effect.

Sorafenib is the only effective systemic therapy for the treatment of HCC; however, side effects may lead to treatment discontinuation in certain patients. The described case highlights how, in the case of sorafenib-related side effects, reductions in the administered dose permit long-term treatment. The standard dose of $400 \mathrm{mg}$ twice a day was selected as the maximum tolerated dose (MTD) based on a phase I study by Strumberg et al (9). Whether low-dose therapy is as effective as high-dose therapy is not clear, but this was true in the case of our patient. The efficacy of conventional cytotoxic agents is positively correlated with the administered dose. With new targeted agents, the length of treatment (as opposed to the dose intensity) may be fundamental for tumor control. The most effective strategy may involve managing the sideeffects of treatment and tailoring the anticancer regimen to the characteristics of the patients, rather than discontinuing treatment at the appearance of signs of intolerance. Notably, although the recommended sorafenib dose is $400 \mathrm{mg}$ twice a day, in certain cases, dose reductions that limit the side effects may offer a better quality of life and may allow for long-term administration and maintenance of tumor control. Data concerning the blood drug levels that are required to achieve and maintain target inhibition are inadequate. The multi-target nature of sorafenib is one additional challenge, as it implies that various factors play a role in the activity of this agent (10).

The radiological features of responding lesions also require investigation. When assessing the efficacy of targeted therapies by imaging, a gradual change in tumor density and blood flow may be observed prior to tumor shrinkage. In certain cases, these lesions have been known to respond and become substituted by residual scars (11). However, uncommon radiological patterns may lead to late recognition of responses or even to misleading evaluations. This issue requires serious consideration, along with new, more appropriate methods of appraisal. With targeted therapies, traditional methods of quantitative evaluation, including WHO criteria or Response Evaluation Criteria in Solid Tumors (RECIST), may not be optimal and the requirement for qualitative standardized measurements becomes more imminent. Recently, modified RECIST (mRECIST) were developed based on measurements of viable tumors with arterial enhancement on a computed tomography (CT) scan, which should be used for the standard assessment of treatment efficacy, particularly in patients who are receiving antiangiogenic drugs (12). Moreover, functional 18 fluorodeoxyglucose (18FDG)-positron emission tomography (PET) imaging provides an additional tool to assess tumor activity. The integration of 18FDG-PET, MRI and CT allows for more precise characterization of drug responses in this disease, which enhances the evaluation of oncologic patients treated with molecularly targeted drugs and accelerates drug development in numerous types of tumors $(13,14)$. In the described case, lesions appeared to be unchanged and would initially have been considered to be active. However, abnormal enhancement lesions disappeared following treatment, so these lesions may have been substituted by residual scars.

To our knowledge, although sorafenib has proven effective in prolonging survival of advanced or unresectable HCC in numerous clinical trials, there are currently no reported cases of long-term progression-free survival for five years. In our case, the patient's follow-up CT/PET and MRI scans demonstrated that no new lesions were present in the body and the AFP serum level has remained within the normal range for almost five years, thus demonstrating progression-free survival. This is the first documented case of an advanced HCC patient with bone metastasis who achieved long-term progression-free survival due to the administration of a reduced dose of sorafenib. The duration of overall survival and the time to progression of the patient have been significantly prolonged, his quality of life has been elevated and no markedly adverse side-effects are evident.

In summary, these data suggest that sorafenib may be a well-tolerated treatment option, even in cases where the tumor has spread extrahepatically. This unexpected outcome raises certain questions and may become a matter of debate. For example, it may be assumed that in tumor cells, mutations or alterations of the genomic profile that cause the cells to become more sensitive to sorafenib may have occurred. If this hypothesis is true, it represents a valid reason to increase new investigations aiming to study in-depth further genetic and molecular features in order to identify more selective (or even individual) patterns of response.

\section{References}

1. Bosch FX, Ribes J, Cléries R, et al: Epidemiology of hepatocellular carcinoma. Clin Liver Dis 9: 191-211, 2005.

2. Llovet JM and Bruix J: Novel advancements in the management of hepatocellular carcinoma in 2008. J Hepatol 48 Suppl 1: S20-37, 2008

3. Gish RG, Porta C, Lazar L, et al: Phase III randomized controlled trial comparing the survival of patients with unresectable hepatocellular carcinoma treated with nolatrexed or doxorubicin. J Clin Oncol 25: 3069-3075, 2007.

4. Cheng AL, Kang YK, Chen Z, et al: Efficacy and safety of sorafenib in patients in the Asia-Pacific region with advanced hepatocellular carcinoma: a phase III randomised, double-blind, placebo-controlled trial. Lancet Oncol 10: 25-34, 2009.

5. Llovet JM, Ricci S, Mazzaferro V, et al: Sorafenib in advanced hepatocellular carcinoma. N Engl J Med 359: 378-390, 2008.

6. Yoshida T, Hisamoto T, Akiba J, et al: Spreds, inhibitors of the Ras/ERK signal transduction, are dysregulated in human hepatocellular carcinoma and linked to the malignant phenotype of tumors. Oncogene 25: 6056-6066, 2006.

7. Huitzel-Melendez FD, Saltz LB, Song J, et al: Retrospective analysis of outcome in hepatocellular carcinoma (HCC) patients with hepatitis $\mathrm{C}(\mathrm{C}+)$ versus $\mathrm{B}(\mathrm{B}+)$ treated with sorafenib (abstract). Presented at the 2007 ASCO Gastrointestinal Cancers Symposium. (abstract 173), Orlando, FL, 2007. 
8. Zhu AX: Predicting the response to sorafenib in hepatocellular carcinoma: where is the evidence for phosphorylated extracellular signaling-regulated kinase (pERK)? BMC Med 7: 42, 2009.

9. Strumberg D, Richly H, Hilger RA, et al: Phase I clinical and pharmacokinetic study of the Novel Raf kinase and vascular endothelial growth factor receptor inhibitor BAY 43-9006 in patients with advanced refractory solid tumors. J Clin Oncol 23: 965-972, 2005

10. Petrelli A and Giordano S: From single- to multi-target drugs in cancer therapy: when aspecificity becomes an advantage. Curr Med Chem 15: 422-432, 2008.

11. Abbadessa G, Rimassa L, Pressiani T, et al: Optimized management of advanced hepatocellular carcinoma: four long-lasting responses to sorafenib. World J Gastroenterol 17 : 2450-2453, 2011.
12. Edeline J, Boucher E, Rolland Y, et al: Comparison of tumor response by Response Evaluation Criteria in Solid Tumors (RECIST) and modified RECIST in patients treated with sorafenib for hepatocellular carcinoma. Cancer 118: 147-156, 2012.

13. Milano A, Perri F, Ciarmiello A, et al: Targeted-therapy and imaging response: a new paradigm for clinical evaluation? Rev Recent Clin Trials 6: 259-265, 2011.

14. Horger M, Lauer UM, Schraml C, et al: Early MRI response monitoring of patients with advanced hepatocellular carcinoma under treatment with the multikinase inhibitor sorafenib. BMC Cancer 9: 208-209, 2009. 